\title{
Notfallmedizinische Kommunikation im Unterricht Polnisch als Fremdsprache. Analyse von Gesprächsverhalten deutschsprachiger Rettungskräfte
}

\author{
Emergency medical communication \\ in Polish as a foreign language classes. \\ Analysis of conversation strategies \\ of German paramedics
}

\begin{abstract}
This paper focuses on the emergency communication of German paramedics who are learning Polish as a foreign language. This is to be tested in a role play, in which speech acts occur. Can the recorded speech acts or certain pragmatic phenomena be used in foreign language teaching of emergency communication? The study shows that the majority of patients' responses are understood by the paramedics, but they respond to them inappropriately. Therefore, it is concluded that language courses for paramedics should focus much more on fixed phrases in Polish. The analysis of the recordings shows that this would enable them to formulate their answers in Polish, and not in German or English. In addition, code-switching sequences were used as means to identify the areas where there is a need for a modification of the programme and exercises during the lessons. The emphasis should lie on non-medical answers to the patient's linguistic actions as well.
\end{abstract}

KEYWORDS: emergency medical communication, Polish as a foreign language, linguistic performing speech acts in medical emergency.

SCHLÜSSELWÖRTER: Notfallkommunikation, Polnisch als Fremdsprache, sprachliches Handeln im medizinischen Notfall.

Medizin und Angewandte Linguistik sind enger verwoben als viele annehmen, insbesondere im Falle eines medizinischen Notfalls. Sprache, genauer gesagt Kommunikation in einer Fremdsprache, kann Leben retten. Dieser Artikel thematisiert Kommunikationsabläufe zwischen den Rettungskräften (Arzt, 
Notfallsanitäter, Rettungsassistent ${ }^{1}$ ) sowohl mit Patienten und / oder deren Familie als auch untereinander im medizinischen Team. Im Fokus des Beitrags stehen Gespräche mit Patienten, die in lebensbedrohlichen Situationen in einer für die Rettungskraft als Fremdsprache gelernten Sprache (hier: auf Polnisch) stattfinden. Die notfallmedizinische Kommunikation ${ }^{2}$ wird also gezielt aus fremdsprachendidaktischer Perspektive betrachtet. Dazu wird auf Daten aus einem Projekt (InGRiP) zurückgegriffen, in dem das Fremdsprachenlernen für die Notfallkommunikation und für die Bewältigung lebensbedrohlicher Lagen eine zentrale Rolle einnimmt.

Mit Blick auf den Themenbereich Fremdsprachlernen für die Notfallkommunikation stellen sich nun die folgenden Forschungsfragen: Welche Sprechhandlungen werden in Notfallsituation vollzogen und welche konkreten Sprechakte kommen vor bzw. müssen von den Fremdsprachenlernenden beherrscht werden, um die Situation kommunikativ erfolgreich zu meistern? Inwiefern können die vorkommenden Sprechhandlungen dafür genutzt werden, zielgerichtete Programme für den Fremdspracherwerb in der Notfallkommunikation zu entwickeln?

\section{HINTERGRUND: DAS PROJEKT INGRIP}

Das Projekt „Integrierter grenzüberschreitender Rettungsdienst Pomerania / Brandenburg" (InGRiP) 3 ist ein aus den Mitteln des Fonds für Regionale Entwicklung (EFRE) finanziertes Projekt, dessen fremdsprachendidaktische und kommunikationswissenschaftliche Module am Institut für Slawistik der Universität Greifswald bearbeitet werden. Im Allgemeinen geht es in diesem Projekt um die transnationale notfallmedizinische Versorgung der Patienten im deutsch-polnischen Grenzraum. Die Miteinbeziehung der Nachbarsprache ${ }^{4}$ in die Tätigkeit der Notfalldienste macht dahingehend Sinn, dass die Patienten in

${ }^{1}$ Aus Gründen der besseren Lesbarkeit wird im Text verallgemeinernd das generische Maskulinum verwendet. Diese Formulierungen umfassen gleichermaßen weibliche, männliche oder diverse Personen. Alle sind damit selbstverständlich gleichberechtigt angesprochen.

${ }^{2}$ Die im Text vorkommenden Begriffe notfallmedizinische Kommunikation und Notfallkommunikation werden synonymisch verwendet.

${ }^{3}$ Nähere Informationen zum Projekt, das in den Jahren 2017-2020 realisiert wird, sowie zu den beteiligten Partnern finden Sie unter https://www.medizin.uni-greifswald.de/ingrip/de/ home/ [Zugriff am: 20.02.2020].

${ }^{4}$ Nachbarsprache ist ein im sprachwissenschaftlichen Diskurs eher selten verwendeter Begriff, der einer näheren Abgrenzung zum Begriff Fremdsprache, insbesondere in einer Grenzregion, bedürfte, vgl. Brehmer (2018). Einen genaueren Versuch der Definition dieses Begriffs nehmen Hryniewicz und Lisek (2019) vor. 
der Grenzregion eine Fremdsprache sprechen und die Rettungskräfte, die sie behandeln, aus dem Ausland kommen. Somit wird die fremdsprachliche Kommunikation mit den Patienten sowie die Arbeit in binationalen Notfallteams ein zentrales Thema des Vorhabens. Im Projekt wurden daher unter anderem fachsprachliche, berufsbezogene Sprachschulungen sowohl auf Polnisch (für die deutschen Rettungskräfte) als auch auf Deutsch (für ihre polnischen Pendants) entwickelt und zunächst für beide Gruppen getrennt durchgeführt. ${ }^{5} \mathrm{Um}$ die Strategien der Kommunikation in Notfallsituationen in der jeweiligen Nachbarsprache möglichst authentisch zu trainieren, nehmen Rettungssanitäter und Notärzte aus beiden Ländern an einem abschließenden gemeinsamen Simulationstraining teil. Dabei liegt das sprachwissenschaftliche Augenmerk im Projekt auf der Erfassung und Auswertung von Problemen in der deutsch-polnischen notfallmedizinischen Kommunikation, sowohl im Hinblick auf sprachliche als auch auf interkulturelle Herausforderungen.

\section{NOTFALLMEDIZINISCHE KOMMUNIKATION}

Kommunikation ist ein sozialer Prozess, in dem verbale oder nonverbale Elemente eine Rolle spielen. Medizinische Kommunikation als eine Form von Kommunikation wird in diesem Artikel bewusst als funktionale Gesprächssituation bezeichnet, d.h. das Gespräch zwischen den Akteuren hat eine konkrete Funktion - hier eine adäquate medizinische Patientenversorgung. Medizinische Kommunikation ist ein vielschichtiger Begriff, wobei es an dieser Stelle nicht das Ziel sein soll, ihn abschließend oder auch nur annähernd zu definieren. Sie ist wie andere Gesprächssituationen „in ein Netz wechselseitiger Kommunikationshandlungen eingebettet" (Bechmann 2014: 6), in denen typische sprachliche Handlungsmuster identifiziert werden können. Von Bedeutung sind dabei die Akteure des Gesprächs sowie der situative Kontext. Bechmann (2014: 2) weist darüber hinaus auf die allgemein spezifische Rolle medizinischer Kommunikation hin, die vor allem medizinisches Handeln bedeute. Die hier thematisierte medizinische Kommunikation findet in der Regel außerhalb des Krankenhauses bei Patienten zu Hause oder an einer Unfallstelle statt. An dieser Kommunikation nehmen Notfallsanitäter, Notärzte, Patienten, Angehörige der Patienten oder Zeugen teil. Die Notfallkommunikation fokussiert also medizinisches und sprachliches Handeln in einer potenziell lebensbedrohlichen Situation. Die in diesem Artikel thematisierte medizinische Kommunikation hat eine zusätzliche Dimension, nämlich die Interkulturalität. Die Notfallkommunikation stellt im

\footnotetext{
${ }^{5}$ In der weiteren Beschreibung des Schulungskonzepts wird nur auf das Polnische als Fremdsprache eingegangen.
} 
grenzüberschreitenden Rettungsdienst zugleich interkulturelle Kommunikation dar. Dies ist insbesondere für Patientenversorgung wichtig, worauf Azizi und Golsabahi-Broclawski (2020: 120) hinweisen: „Missverständnisse, die auf mangelhafte Kenntnisse der Kultur des Gegenübers basieren, können in der Notfallmedizin für den betroffenen Patienten tödliche Folgen haben." Speziell im deutsch-polnischen Kontext ist zu bemerken, dass z.B. der Umgang mit Krankheit und Tod kultursensibel erfolgt. Wichtig erscheint dabei die Sensibilisierung um die berufsbezogenen Befugnisfragen: Die Rettungssanitäter haben auf beiden Seiten der Grenze unterschiedliche Befugnisse bezüglich der Durchführung medizinischer Maßnahmen. ${ }^{6}$

Je nach medizinischem Notfall und Gesprächspartner (Patient, Angehöriger, Mitglied des Rettungsteams) weist ein solches Gespräch mehr oder weniger starke Elemente einer Fachkommunikation auf. Dabei kommt der Verständigung die oberste Priorität zu, was bereits für herkömmliche Arzt-Patienten-Gespräche, beispielsweise in einer Arztpraxis oder im Krankenhaus, gilt: „Eine gelungene Verständigung zwischen Arzt und Patient ist die Voraussetzung für eine gelungene medizinische Versorgung, denn die Arzt-Patient-Kommunikation ist der Ausgangspunkt für eine konkrete Diagnose, eine abgestimmte Behandlung und einen effektiven Therapieverlauf" (Spranz-Fogasy \& Becker 2015: 93). Dies ist noch mehr der Fall, wenn es sich um einen Notfall handelt (z.B. Herzinfarkt). Die Situation wird in unserem Fall für die Rettungskräfte zusätzlich dadurch verschärft, dass die Gespräche auf Polnisch stattfinden, d.h. in einer Fremdsprache. Die Fremdsprache (und ihr gutes Erlernen) spielt somit eine wichtige Rolle. Nicht weniger wichtig ist die Beziehungsebene in dem Gespräch (vgl. von Thun 1998: 30) oder die Tatsache, dass die Kommunikation in der Regel asymmetrisch ${ }^{7}$ verläuft (vgl. Kiesendahl 2011: 41). Emotionen wie Angst, Aufregung sowie Schmerz oder Ärger können ebenso ein Faktor sein, der den Gesprächsverlauf beeinflusst. Darüber hinaus ,[sind] Notfallpatienten aufgrund ihrer speziellen Lage häufig weder ausreichend auf das ärztliche Gespräch vorbereitet noch fähig, zielführend zu kommunizieren“" (Bechmann 2014: 176).

In der Notfallkommunikation wird mit Schemata ${ }^{8}$ zur Notfallanamnese wie SAMPLER (u.a. zur Feststellung von Symptomen, Allergien und Vorerkran-

${ }^{6}$ Die polnischen Rettungssanitäter dürfen aufgrund ihres Bachelorabschlusses den Patienten andere Medikamente als ihre deutschen Kollegen verabreichen. Hieraus bildet sich eine interkulturelle Herausforderung, auf welche die Kursteilnehmenden sensibilisiert werden müssen.

${ }^{7}$ Dabei ist sowohl eine fachlich-sachliche als auch eine gesprächsstrukturelle Asymmetrie gemeint, d.h. die Rettungskräfte haben einen Wissensvorsprung gegenüber den Patienten und ihnen obliegt die Gesprächsführung in der Notfallkommunikation.

8 „Um eine schnelle Übersicht an einem Unfallort oder Vorfallort, v. a. im außerklinischen Setting, zu erreichen, können [...] Akronyme angewendet werden“" (Stemmler \& Hecker 2017: 100), z. B. ABCDE oder SAMPLER. Diese werden auch Schemata zur Notfallanamnese genannt. Unter 
kungen) und OPQRST (u.a. zur Feststellung der Art, Stärke und dem Verlauf der Beschwerden) gearbeitet. Bei der Patientenübergabe im Krankenhaus sind ATMIST oder SBAR üblich, die beispielsweise die Krankheitssymptome, den Verletzungsmechanismus (z.B. Sturz) oder bereits eingeleitete Maßnahmen in den Fokus stellen. Die Notfallschemata unterscheiden sich im Wesentlichen von der nicht vom Notfall bedingten medizinischen Arzt-Patient-Kommunikation durch ihre Kürze und enorme Fokussierung. Die medizinischen Schemata, die sich im alltäglichen Arzt-Patient-Gespräch in einer Arztpraxis oder im Krankenhaus als defizitär erweisen (vgl. Bechmann 2014: 170), gelten während einer medizinischen Notfallkommunikation als Abhilfe bei Anamnese und Exploration. Die Notfallschemata sind nicht nur ein Werkzeug für eine schnelle und an den Beschwerden orientierte Notfallanamnese bzw. Patientenübergabe, sondern bestehen auch aus konkreten Sprechhandlungen, die sich aus einzelnen Sprechakten wie z.B. Verlangen, Befehlen, Beschreiben zusammensetzen (vgl. Searle 1997: 39). Angesichts der Spezifika der Notfallkommunikation und des Vorliegens fester Schemata zur Anamnese bzw. Patientenübergabe erscheint das gezielte Erlernen ausgewählter Äußerungen in Intensivkursen fremdsprachendidaktisch produktiv und sinnvoll, um einen schnellen Wissenszuwachs zu gewährleisten und situationsadäquates Handeln in der Fremdsprache zu sichern. Für die Teilnehmenden kann es darüber hinaus auch spannend sein, zu sehen, wie die Realisierung der notfallmedizinischen Schemata in der Fremdsprache aussieht. Folglich wurde in den Intensivkursen des Polnischen im Projekt InGRiP das adäquate kommunikative Verhalten in Notfallsituationen schwerpunktmäßig anhand ausgewählter Fallszenarien eingeübt, deren allgemeiner Ablauf den Teilnehmenden aus der eigenen Berufspraxis in Deutschland bekannt war.

Bevor näher auf die in Notfallsituationen zu erwartenden Sprechakte eingegangen wird, soll zunächst ein Notfallgespräch in Bezug auf dort anzutreffende Fragetypen charakterisiert werden. In Anlehnung an Spranz-Fogasy und Becker (vgl. 2015: 100) ist zu konstatieren, dass auch in der Notfallkommunikation Fragen das erste Arbeitswerkzeug des Notfallsanitäters sind.

Die Fragetypen, die für medizinische Kommunikation charakteristisch sind, wurden bereits von Bechmann klassifiziert und beschrieben (vgl. Bechmann 2014: 190ff.). Daraus lassen sich für die Notfallkommunikation folgende Fragetypen ableiten, die zur Veranschaulichung mit Beispielen versehen werden:

den Schemata der Notfallanamnese sind Fragenkataloge oder eine festgelegte Abfolge von Fragen gemeint, dank deren gezielt einzelne Informationen eingeholt werden können. Mögliche Fragen des SAMPLER-Schemas lauten wie folgt: (S) Welche Beschwerden haben Sie jetzt? (A) Haben Sie Allergien? (M) Nehmen Sie Medikamente? (P) Haben Sie Vorerkrankungen? (L) Wann haben Sie das letzte Mal gegessen? (E) Was haben Sie direkt vor dem Auftreten der Beschwerden gemacht? (R) Rauchen Sie? Haben Sie Diabetes? 
a) Informationsfragen

Co teraz panu / pani dolega?

Was fehlt Ihnen jetzt?

b) Einschätzungsfragen

Na skali od 0 do 10, jak silny to ból?

Auf der Skala von 0 bis 10, wie stark ist der Schmerz?

c) Verhaltensfragen

Brat(a) pan(i) dziś już leki?

Haben Sie heute schon ihre Medikamente eingenommen?

d) Kettenfragen

Dlaczego i kiedy był(a) pan(i) ostatni raz w szpitalu?

Wann waren Sie das letzte Mal im Krankenhaus und warum?

Die Fragen gehören einer übergeordneten Einheit, nämlich zum Sprechhandeln. Die folgenden Sprechhandlungen auf Polnisch sind aufgrund der medizinischen Tätigkeit zu erwarten:

a) seitens der Rettungskräfte

- Vorstellung,

- Fragen nach allgemeinen Daten des Patienten,

- spezifische Fragen zum Gesundheitszustand, Beschwerden und Allergien,

- Fragen nach möglichen ähnlichen Vorfällen in der Familie,

- Erklärungen zum Zustand des Patienten sowie zum weiteren medizinischen Vorgehen.

b) seitens des wachen Patienten oder eines Zeugens oder eines / einer Angehörigen

- Vorstellung,

- Klagen über die Beschwerden, ihre Intensität, Verortung und Dauer,

- Informieren über den allgemeinen Gesundheitszustand und Allergien,

- Informieren über ähnliche Vorfälle in der Familie,

- Fragen nach der Einschätzung des Zustands sowie zum weiteren medizinischen Vorgehen.

Die Sprechhandlungen seitens der Rettungskräfte lassen sich weiter danach einteilen, ob sie vom ärztlichen oder nicht-ärztlichen Personal durchgeführt werden. Die thematisierten Sprechhandlungen sind Gegenstand der Unterrichtseinheiten im Sprachkurs. 
Nachdem die Sprechhandlungen und die dazugehörigen Fragetypen betrachtet worden sind, wird das Augenmerk auf eine untergeordnete Ebene gelenkt, d.h. zu Sprechakten. Aus linguistischer Perspektive sind in der Notfallkommunikation folgende Sprechakte zu erwarten:

a) Assertiva, wenn Ärzte oder Rettungskräfte über den Patientenzustand berichten oder wenn der Patient über die Beschwerden sachlich informiert;

b) Direktiva, wenn Ärzte oder Rettungskräfte um etwas bitten, etwas fragen, zu etwas auffordern oder wenn etwas dem Rettungsteam oder Patienten befohlen wird;

c) Kommissiva, wenn Ärzte oder Rettungskräfte ein Vorgehen untereinander oder mit dem Patienten vereinbaren. Denkbar wäre auch eine Bitte des Notarztes an den Patienten zur Mitarbeit bei der Behandlung oder Drohungen seitens eines aggressiven Patienten gegenüber dem Rettungsteam;

d) Expressiva, wenn Ärzte oder Rettungskräfte die Patienten grüßen oder die Patienten über Schmerzen klagen.

\section{LEHRKONZEPT ZUR NOTFALLMEDIZINISCHEN KOMMUNIKATION IM POLNISCHEN ALS FREMDSPRACHE}

Die Abbildung veranschaulicht den Ablauf und die Komponenten der notfallmedizinisch ausgerichteten Sprachschulung im Polnischen als Fremdsprache. ${ }^{9}$

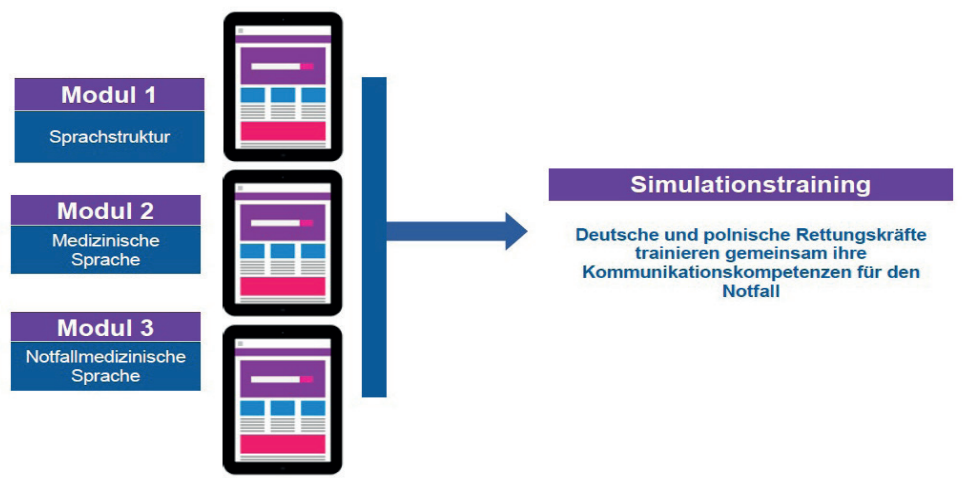

Abbildung 1. Bestandteile des sprachlichen und interkulturellen Schulungskonzepts

${ }^{9}$ Um eine Diskussion der Ziele der Fachsprachdidaktik bewusst nicht zu entfachen, wurde hier die Bezeichnung Polnisch als Fremdsprache benutzt, auch wenn fachsprachendidaktische Ziele im Kurs verfolgt werden. Dies ist zu einem der sprechakttheoretischen Ausrichtung des Artikels geschuldet, zu anderem konnten zu Beginn des Projektes nicht die fachsprachliche Bedarfsanalyse im vollen Umfang durchgeführt werden, wie die in der einschlägigen Literatur gefordert wird (vgl. Gębal 2016: 27). 
Das Schulungskonzept sah sowohl Präsenzveranstaltungen als auch E-Learning-Phasen vor. Die Präsenzveranstaltungen (Module 1-3) dauerten jeweils eine Woche pro Modul, wobei die Blöcke nicht direkt aufeinander folgten, sondern über das Jahr 2019 verteilt waren.

Im ersten Modul wurde eine Einführung in elementare sprachliche Strukturen des Polnischen vermittelt (Aussprache, Grammatik ${ }^{10}$ ). Im zweiten Modul rückte die Vermittlung medizinischer Terminologie (insb. Lexik zu den biologischen Systemen des Körpers und ihre Krankheitssymptomatik) in den Mittelpunkt. Sowohl im ersten als auch im zweiten Modul wurden bereits auch speziell notfallmedizinische Fragen und Äußerungen eingeübt, die dann im dritten Modul den thematischen Fokus bildeten, wobei - wie bereits erwähnt - die etablierten Schemata der Notfallrettung und Patientenübergabe die Leitschnur für die einzelnen Sitzungen abgaben.

Der Präsenzkurs umfasste somit insgesamt 120 Einheiten ${ }^{11}$ pro Teilnehmenden. Der Umfang der Präsenzkurse war größtenteils den zeitlichen Beschränkungen und Arbeitsbelastungen der Rettungskräfte geschuldet. Insbesondere die organisatorisch und finanziell aufwändige Freistellung der Kursteilnehmenden ließ keinen höheren Umfang der Präsenzschulung zu. Um dennoch eine kontinuierliche Beschäftigung der Kursteilnehmenden mit der Fremdsprache zu ermöglichen, wurden bewährte Verfahren des Blended-Learnings (vgl. Zschäbitz 2013; Szerszeń 2014) in das Schulungsprogramm integriert. Es wurden E-Learning-Module entwickelt, die in der Zeit zwischen den Präsenzschulungen von den Kursteilnehmenden selbstständig bearbeitet werden konnten. Pro E-Learning-Modul waren insgesamt 25 Stunden Workload vorgesehen. Die ersten zwei Einheiten gingen jeweils über sieben Wochen, die letzte Einheit erstreckte sich über acht Wochen. Den Abschluss der Schulung bildet ein gemeinsames Simulationstraining ${ }^{12}$ der deutschen und polnischen Kursteilnehmenden, in dem die in den Kursen vermittelten sprachlichen Strukturen und das interkulturelle Wissen in einer Face-to-Face-Situation in gemischten Gruppen angewendet werden sollen.

${ }^{10}$ Da es sich um einen Intensivsprachkurs handelt, können im Rahmen von drei Wochen nur gewisse grammatische Strukturen vermittelt werden. Dazu gehören u.a. Verbflexionsformen in Präsens, ausgewählte Formen des Präteritums. Deklination der Substantive und Adjektive in ausgewählten Fällen (Nom., Dat., Akk., Instr.) im Singular. Einführung in Präpositionen, Adverbien sowie Modal- und Zahlwörter war ebenso Teil des Kurses.

${ }^{11}$ Als Einheit gelten 45 Minuten.

${ }^{12}$ Die Coronakriese hat den Abschluss des Simulationstrainings verzögert, d.h. die Trainingseinheiten sollen in Oktober und November statt in Mai 2020 abgeschlossen werden. 


\section{DATEN UND METHODE}

Um das Gesprächsverhalten deutschsprachiger Rettungskräfte analysieren zu können, wurden Sprachdaten gesammelt. Die vorliegenden Sprachdaten wurden im Rahmen eines (als Test konzipierten) offenen, mündlichen Rollenspiels (Kurzsimulation $^{13}$ ) während der dritten Woche des Polnischunterrichts für deutsche Rettungskräfte zwischen August und Oktober 2019 in Greifswald erhoben. Die Kursteilnehmenden sollten sich dabei vorstellen, dass sie an einem Einsatzort eintreffen und dort ein Anamnesegespräch mit einem muttersprachlichen (polnischsprachigen) Patienten durchführen. Die Lehrperson gab den Probanden keine Zeit für die Vorbereitung des Dialogs, d.h. die teilnehmenden Rettungskräfte hatten keine Zeit, Frage-Antwort-Sequenzen oder die Reihenfolge ihrer Äußerungen zu planen, um möglichst das freie Ad-hoc-Formulieren und damit eine Situation, wie sie für authentische Face-to-Face-Interaktionen typisch ist, analysieren zu können.

Die Testaufgabe bestand konkret darin, ein Notfallanamnesegespräch mit Fragen zu Symptomen, Allergien, Vorerkrankungen, letzten Mahlzeiten, Ereignissen vor dem Notfall und individuellen Risikofaktoren durchzuführen. Des Weiteren sollten die Probanden Informationen zum Beginn der Beschwerden sammeln, die Stärke und den Charakter des Schmerzes erfragen, Informationen zum Schmerzverlauf erheben sowie den Schmerz verstärkende oder lindernde Faktoren erfassen. Untersucht wurde dabei, ob und inwieweit die Kursteilnehmenden die in den Notfallanamnese-Schemata vorgesehenen Äußerungen in der Fremdsprache verinnerlicht haben.

Einerseits sind Antworten bei offenen Testverfahren nicht gänzlich vorhersehbar (vgl. Albert \& Koster 2002: 100), andererseits werden in der Notfallkommunikation etablierte medizinische Notfallschemata angewandt. Diese garantieren ein gewisses Maß an Vorhersehbarkeit. Der hier eingesetzte offene Test ermöglicht des Weiteren die Sprechhandlungsmuster, die deutsche Rettungskräfte in polnischer Sprache für die Notfallkommunikation anwenden (können), nachzuzeichnen.

Die Fallbeispielanalyse erfolgt anhand einer Aufnahme, die in einem Gruppensetting aufgezeichnet wurde. Das ganze Gespräch findet zwischen dem Patienten (Piotr Nowak - PN) ${ }^{14}$, dem leitenden Notfallsanitäter (Klaus Kleber KK), dem Notarzt (NA) und weiteren Notfallsanitätern (NS2, NS3, NS4) statt, die einzelne Fragen ergänzen. Die Rettungskräfte wurden zu einem an Atemnot

${ }^{13}$ Über Funktionen, Bedeutung und Vorteile von Simulationen im Sprachunterricht berichten Illés und Kegyes (vgl. 2019) zur Fachsprache für Logistiker sowie Klčová (vgl. 2019) zur Fachsprache für Juristen.

${ }^{14}$ Die Rolle des Patienten wurde in dem Rollenspiel von der muttersprachlichen Lehrkraft des Polnischen übernommen, um gewährleisten zu können, dass die Probanden nur mit der Lexik konfrontiert werden, die bereits im Unterricht vermittelt worden war. 
leidenden Patienten gerufen. Das Gespräch dauerte insgesamt 5 Min. 21 Sek und wurde mit dem Partitur-Editor von EXMARaLDA ${ }^{15}$, einem Transkriptionssystem für mündliche Korpora, in der HIAT-Konvention ${ }^{16}$ transkribiert.

\section{ANALYSE DER SPRECHHANDLUNGEN}

\subsection{Makrostruktur des Gesprächs und Fragetypen zum Thema Atemnot}

In diesem Abschnitt soll die Makrostruktur des notfallmedizinischen Gesprächs zum Thema Atemnot mit dazugehörigen Fragen beleuchtet werden. Im Fokus stehen Sprechhandlungen, die durch einzelne Sprechakte seitens des Rettungsteams oder des Patienten realisiert werden.

$[1]$

\begin{tabular}{|c|c|c|c|c|}
\hline & 0 [00:00.0] & 1 [00:02.0] & 2 [00:02.7] & 3 [00:07.5] \\
\hline KK [v] & Dzień dobry! & & $\begin{array}{l}\text { e Nazywam się } \\
\text { Klaus Kleber. }\end{array}$ & \\
\hline $\mathrm{PN}$ [v] & & Dzień dobry! & & \\
\hline NS2 [v] & & & & $\begin{array}{l}\text { ((Lachen im Hinter- } \\
\text { grund }))\end{array}$ \\
\hline translat & Guten Tag! & Guten Tag! & $\begin{array}{l}\text { Ich heiße Klaus } \\
\text { Kleber. }\end{array}$ & \\
\hline
\end{tabular}

[2]

\begin{tabular}{|c|c|c|c|c|c|c|c|}
\hline & 4 [00:09.5] & 5 [00:10.7] & 6 [00:11.2] & 7 [00:14.9] & 8 [00:16.4] & 9 [00:19.1] & 10 [00:19.3] \\
\hline KK [v] & $\begin{array}{l}\text { Geht's Dir } \\
\text { gut? }\end{array}$ & & $\begin{array}{l}\text { Jestem ee } \\
\text { ratowni- } \\
\text { kem }\end{array}$ & & & & $\mathrm{Co} ?$ \\
\hline PN [v] & & & & $\begin{array}{l}\text { Dzień } \\
\text { dobry. }\end{array}$ & $\begin{array}{l}\text { Piotr } \\
\text { Nowak. }\end{array}$ & Dzień dobr... & \\
\hline NS2 [v] & & Ja! & & & & & \\
\hline translat & & & $\begin{array}{l}\text { Ich bin Ret- } \\
\text { tungssan- } \\
\text { itäter. }\end{array}$ & Guten Tag. & & Tag & Was? \\
\hline
\end{tabular}

${ }^{15}$ Mehr zu EXMARaLDA und den Funktionalitäten des Transkriptionssystems finden Sie auf der Projekt-Webseite: https:/ / exmaralda.org/de/ueber-exmaralda/ [Zugriff am: 11.11.2019].

${ }^{16}$ Die Wahl der Transkriptionskonvention erfolgt aus forschungspraktischen Zwecken und resultiert vor allem aus ihrer Eignung zur Erfassung gleichzeitiger Gesprächsbeiträge vieler Gesprächsteilnehmer (vgl. Mroczynski 2014: 54), was in einem so konzipierten Gruppensetting nicht auszuschließen ist. 
[3]

\begin{tabular}{|l|lll|}
\multicolumn{1}{l|}{11 [00:20.3] } & $12[00: 23.0]$ & $13[00: 28.2]$ \\
KK [v] & y Co Pana boli? & & y Jak sylny to \\
translat & Was tut Ihnen weh? & $\begin{array}{l}\text { yuszności. } \\
\text { dier tut's weh. Hier hier tut's mir weh. Ich } \\
\text { habe Atemnot. }\end{array}$ & Wie stark (sic!) \\
\hline
\end{tabular}

Der Rettungssanitäter markiert die Eröffnungsphase, indem er sich vorstellt und den Patienten (Z. 1) begrüßt. Des Weiteren fragt Klaus Kleber, was dem Patienten wehtut (Zn. 2-3) und eröffnet somit die Kernphase des Gesprächs, in der er mehrere Fragen zu den Beschwerden und ihrer Intensität stellt.

In der Kernphase dominieren Informationsfragen (Zn. 2-3, 10, 11,12, 15, 16, 17, 19, 22, 22-23, 23-24), die für die Diagnose und Planung der notfallmedizinischen Therapiemaßnahmen nötig sind. Die genannten Fragen seitens der Rettungskräfte stellen folgende Sprechhandlungen dar: Erfragen möglicher Allergien, Medikamenteneinnahme und Vorerkrankungen, Erfragen eines vergangenen Krankenhausbesuchs. Die Anamnese beinhaltet zwar feste Themenbereiche, die abgefragt werden sollten, aber die Praxis und somit eine konkrete Notfallkommunikation stellen immer einen Einzelfall dar. Grundsätzlich ist es sinnvoll, ein Fallbeispiel $\mathrm{zu}$ analysieren und Sprechhandlungen für das Thema Atemnot zu umrahmen, um die hier erfassten Äußerungen in ihrer kontextgebundenen Ausprägung zu betrachten.

[18]

\begin{tabular}{|c|c|c|}
\hline & $54[03: 43.0]$ & $55[03: 49.0]$ \\
\hline PN [v] & Biorę, biorę czasami no. Biorę, biorę ibuprofen i paracetamol. & \\
\hline NS4 [v] & & $\begin{array}{l}((6,5 \mathrm{~s})) \text { Proszę } \\
\text { maska }\end{array}$ \\
\hline translat & $\begin{array}{l}\text { Ich nehme, nehme manchmal ein. Ich nehme Ibuprofen und Paraceta- } \\
\text { mol. }\end{array}$ & Bitte Maske \\
\hline
\end{tabular}

[19]

\begin{tabular}{|c|c|c|c|c|c|c|}
\hline & 56 [03:57.1] & 57 [03:59.0] & 58 [04:00.3] & 59 [04:03.5] & 60 [04:07.6] & 61 [04:12.8] \\
\hline KK [v] & & & & & $\begin{array}{l}\text { Mam } \\
\text { astme. }\end{array}$ & \\
\hline PN [v] & & & & $\begin{array}{l}\text { mm Tak, } \\
\text { mam astmę. }\end{array}$ & & \\
\hline NA [v] & $\begin{array}{l}\text { Choruje } \\
\text { pan na... }\end{array}$ & & coś? & & & \\
\hline NS3 [v] & & & & & & hat \\
\hline
\end{tabular}




\begin{tabular}{|c|c|c|c|c|c|c|c|}
\hline & 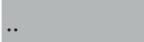 & 56 [03:57.1] & 57 [03:59.0] & 58 [04:00.3] & 59 [04:03.5] & 60 [04:07.6] & 61 [04:12.8] \\
\hline NS4 [v] & tlenowa. & & ptli maska & & & & \\
\hline translat & $\begin{array}{l}\text { mit Sauer- } \\
\text { stoff }\end{array}$ & $\begin{array}{l}\text { Haben } \\
\text { Sie Vorer- } \\
\text { krankun- } \\
\text { gen }\end{array}$ & & was & $\begin{array}{l}\text { Ja, ich habe } \\
\text { Asthma. }\end{array}$ & $\begin{array}{l}\text { Ich habe } \\
\text { Asthma. }\end{array}$ & \\
\hline
\end{tabular}

[20]

\begin{tabular}{|c|c|c|c|}
\hline & 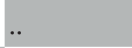 & $62[04: 14.8]$ & $63[04: 24.0]$ \\
\hline PN [v] & & & Gotowałem obiad. Zupę i \\
\hline NA [v] & & $\begin{array}{l}((1,5 \mathrm{~s})) \text { Co pan robił, • zanim ból } \\
\text { się } \bullet \text { pojawił? }\end{array}$ & \\
\hline NS3 [v] & Asthma. & & \\
\hline translat & & $\begin{array}{l}\text { Was haben Sie gemacht, bevor der } \\
\text { Schmerz anfing? }\end{array}$ & $\begin{array}{l}\text { Ich habe Mittagessen gekocht. } \\
\text { Suppe und }\end{array}$ \\
\hline
\end{tabular}

[21]

\begin{tabular}{|c|c|c|c|}
\hline & .. & $64[04: 29.1]$ & 65 [04:32.0] \\
\hline PN [v] & Spaghetti. & & $\begin{array}{l}\text { Gotowałem obiad. } \cdots \cdot \text { mh } \\
\text { Gotowałem obiad. }\end{array}$ \\
\hline NS4 [v] & & Spaghetti hat er gegessen. & \\
\hline translat & Spaghetti Bolognese & & $\begin{array}{l}\text { Ich habe Mittagessen ge- } \\
\text { kocht. }(2 x)\end{array}$ \\
\hline
\end{tabular}

[22]

\begin{tabular}{|c|c|c|c|c|c|}
\hline & 66 [04:36.6] & 67 [04:37.3] & 68 [04:37.8] & 69 [04:41.6] & $70[04: 47.0]$ \\
\hline PN [v] & & & & $\begin{array}{l}\text { Trochę, trochę, } \\
\text { tak, no tak. }\end{array}$ & \\
\hline NA [v] & & & $\begin{array}{l}\text { Czy ból promie- } \\
\text { niuje? }\end{array}$ & & $\begin{array}{l}((2 s)) \text { Czy teraz } \\
\text { boli }\end{array}$ \\
\hline NS3 [v] & & $\mathrm{mhm}$ & & & \\
\hline NS4 [v] & Mittag & & & & \\
\hline translat & & & $\begin{array}{l}\text { Strahlt der } \\
\text { Schmerz aus? }\end{array}$ & $\begin{array}{l}\text { Ein wenig, } \\
\text { wenig, ja, naja. }\end{array}$ & Tut es jetzt weh \\
\hline
\end{tabular}

[23]

\begin{tabular}{|c|c|c|c|c|c|}
\hline & 71 [04:52.0] & 72 [04:55.6] & 73 [04:57.0] & 74 [04:59.0] & 75 [05:04.0] \\
\hline PN [v] & $((2 s))$ Tak! & & Aa! Boli. & & \\
\hline NS2 [v] & & & & & $\begin{array}{l}((\text { Geräusch })) \\
\text { Czy }\end{array}$ \\
\hline
\end{tabular}




\begin{tabular}{|lllllll|} 
&.. & 71 [04:52.0] & 72 [04:55.6] & 73 [04:57.0] & 74 [04:59.0] & 75 [05:04.0] \\
NA [v] & bardziej? & & Tak. & & \\
NS3 [v] & & & & $((2 \mathrm{~s}))(($ iro- & \\
& & & & nisch) boli, & \\
& & & boli & \\
translat & mehr? & Ja! & Ja. & Au! Tut weh. & Tut weh,tut & (Frage-Par- \\
& & & & weh. & tikel) \\
\hline
\end{tabular}

[24]

\begin{tabular}{|c|c|c|c|c|c|}
\hline & .. & 76 [05:10.8] & 77 [05:14.0] & 78 [05:19.0] & 79 [05:21.2] \\
\hline PN [v] & & $\begin{array}{l}\text { Nie palę. Nie } \\
\text { palę. }\end{array}$ & & Dziękuję. & \\
\hline NS2 [v] & pali papierosy? & & & & \\
\hline NA [v] & & & $\begin{array}{l}((3 \mathrm{~s})) \text { Dam koś } \\
\text { na ból. }\end{array}$ & & \\
\hline translat & $\begin{array}{l}\text { Rauchen Sie } \\
\text { Zigaretten? }\end{array}$ & $\begin{array}{l}\text { Ich rauche nicht. } \\
(2 x)\end{array}$ & $\begin{array}{l}\text { Ich gebe etwas } \\
\text { (sic!) gegen den } \\
\text { Schmerz. }\end{array}$ & Danke. & \\
\hline
\end{tabular}

Die zweite Gruppe der Fragen bilden in diesem Notfallgespräch die Verhaltensfragen (Zn. 20 sowie 23-24). Es konnte innerhalb der vorliegenden Notfallkommunikation ebenso eine einzelne, sich wiederholende Einschätzungsfrage (Z.3-4 und 8) ausgemacht werden, die typischerweise bei der Einschätzung der Schmerzstärke durch den Patienten vorkommt.

Die Erklärung des Notarztes zum weiteren medizinischen Vorgehen (Z. 24) markiert die (hier kurz ausfallende) Beendigungsphase des Gesprächs.

Aus der fremdsprachendidaktischen Perspektive erscheinen die Fragetypen und ihre konkrete Realisierung bedeutend für die konzeptuelle Arbeit an einem notfallmedizinischen Kursprogramm sowie an den sprachpraktischen Übungen selbst. Das analysierte Fallbeispiel konturiert die Relevanz für das Lehrprogramm der aus wenigen morphosyntaktischen Elementen bestehenden Fragen für die notfallmedizinische Kommunikation. Zum Testbeginn wurde davon ausgegangen, dass Fragen in der Notfallkommunikation allgemein eine wesentliche Rolle spielen. Es ist dahingehend ein Erkenntnisgewinn, dass im Sprachkurs mehrere Varianten von Fragenformulierungen zum gleichen Sachverhalt vorgestellt und geübt wurden. Während des Rollenspiels wurden wiederum dann die kürzesten davon benutzt. Es ist zu vermuten, dass die Lernenden auch die Formulierungen mit der für sie am einfachsten erscheinenden Aussprache gewählt haben. Aus der fremdsprachendidaktischen Perspektive stellen diese Beobachtungen einen Erkenntnisgewinn dar, da bei der Weiter- 
entwicklung des Fachsprachenkurses die Folgegruppen noch zielgerichteter auf ihre fremdsprachigen Einsätze vorbereitet werden können. Diese Feststellung ist auch im Rahmen der Nachhaltigkeit des Schulungskonzepts relevant, da der Unterricht bei den weiteren Gruppen auf Erlernen von elliptischen Formulierungen fokussiert werden kann.

Im analysierten Gespräch kommen ebenso deutlich die Bedeutung der Antworten und die sprachliche Reaktion auf diese sowie die konkreten Gesprächsstrategien und der Umgang mit Kommunikationsproblemen zur Geltung. Die im Transkript vorkommenden Pausen in den Sequenzen der Rettungskraft können auf Unsicherheit bezüglich der erlernten Wendungen hindeuten, wobei sich ebenso auch Sprachwissenslücken abzeichnen. In diesen Momenten tritt die gruppendynamische Komponente zum Vorschein. Die am Rollenspiel Teilnehmenden versuchten, sich gegenseitig zu unterstützen (Z. 5) und bei Bedarf dolmetschten sie ins Deutsche (Z. 13 sowie Z. 22), wenn sie merkten, dass die Rettungskraft die Äußerung des Patienten nicht verstand. Sie formulierten auch Aussagen auf Polnisch für den Kollegen oder sagten fehlende Lexeme vor.

\subsection{Sprechakte in der Notfallkommunikation zum Thema Atemnot}

Im Rahmen der untersuchten Gesprächssituation ${ }^{17}$ wurden an ersten Stelle Assertiva verwendet. Danach folgen Direktiva und Expressiva. Die Expressiva werden in dem Gespräch vor allem vom Patienten produziert: Herr Nowak klagt mehrmals über die Schmerzen (Zn. 5-9, 12, 14, 17) und tut dies mit Nachdruck durchweg über mehrere Sprechsequenzen (Zn. 5-9).

[3]

\begin{tabular}{|l|lll|}
\hline KK [v] & y Co Pana boli? & $12[00: 23.0]$ & $13[00: 28.2]$ \\
PN [v] & & $\begin{array}{l}\text { ym uf Tu mnie boli. Tu tu mnie boli. Mam } \\
\text { duszności. }\end{array}$ & y Jak sylny to \\
translat & Was tut Ihnen weh? $\begin{array}{l}\text { Hier tut es weh. Hier hier tut es weh. Ich } \\
\text { habe Atemnot. }\end{array}$ & Wie stark (sic!) \\
\hline
\end{tabular}

${ }^{17}$ Der Analyse wurden sowohl die Äußerungen des Patienten (Dozent) als auch der Rettungskräfte (Kursteilnehmende) unterzogen, da diese das Gespräch konstituieren, auch wenn der Patient aufgrund der muttersprachlichen Kompetenz mit seinen Redeanteilen das Gespräch dominierte. Die vorhandene oder fehlende Reaktion seitens der Rettungskräfte lassen auf eine Notwendigkeit schließen, die Schulungsübungen zu erweitern, deswegen werden die Redeanteile des Patienten mit untersucht. 
[4]

\begin{tabular}{|c|c|c|}
\hline & .. & $14[00: 45.4]$ \\
\hline KK [v] & zi zero do desjać ziro niet desjać sjlny & \\
\hline PN [v] & & Może Pan powtórzyć ((3.5s)) Może Pan \\
\hline translat & (Russ.) von null bis zehn null zehn stark (sic!) & Können Sie wiederholen Können Sie \\
\hline
\end{tabular}

[5]

\begin{tabular}{|llll|} 
PN [v] & $\begin{array}{l}\text { powtórzyć, bo ja.., boli mnie } \\
\text { no }\end{array}$ & $15[00: 54.9]$ & Proszę powtórzyć. \\
NS2 [v] & & $($ leise) $)$ er versteht Dich nicht \\
translat & $\begin{array}{l}\text { wiederholen, weil ich...,mir } \\
\text { tuts weh eh }\end{array}$ & Bitte wiederholen. \\
\hline
\end{tabular}

[6]

\begin{tabular}{|c|c|c|c|}
\hline & 17 [01:07.6] & 18 [01:09.6] & 19 [01:12.4] \\
\hline KK [v] & ee & Co Pana boli? & \\
\hline PN [v] & & & $\begin{array}{l}\text { Boli? Boli tu mnie klatka piersiowa boli. Dusz- } \\
\text { no mi. • Tu mnie }\end{array}$ \\
\hline translat & & $\begin{array}{l}\text { Was tut Ihnen } \\
\text { weh? }\end{array}$ & $\begin{array}{l}\text { Weh tut? Der Brustkorb tut mir weh. Ich habe } \\
\text { Atemnot. Hier }\end{array}$ \\
\hline
\end{tabular}

[7]

\begin{tabular}{|c|c|c|c|c|c|c|}
\hline & t & 20 [01:18.2] & 21 [01:24.0] & 22 [01:26.0] & 23 [01:26.2] & 24 [01:27.2] \\
\hline KK [v] & & e Jak sylny? & & Jak silny? ja! & & \\
\hline PN [v] & boli. & & A, jak silny? & & A, jak silny! & $\begin{array}{l}\text { A, jak boli tu } \\
\text { tak? Mocno. } \\
\text { Bardzo. }\end{array}$ \\
\hline translat & tuts weh & $\begin{array}{l}\text { Wie stark } \\
\text { (sic!)? }\end{array}$ & $\begin{array}{l}\text { Acha, wie } \\
\text { stark? }\end{array}$ & Wie stark? & Wie stark! & $\begin{array}{l}\text { Acha,wie } \\
\text { tuts weh,ja? } \\
\text { Stark. Sehr. }\end{array}$ \\
\hline
\end{tabular}

[8]

\begin{tabular}{|l|lll|}
\hline KK [v] & 25 [01:31.9] & $26[01: 40.5]$ & 27 [01:45.6] \\
PN [v] & $\begin{array}{l}\text { do ne s ziro do desjać. } \\
\text { • desjąć sylny? }\end{array}$ & djewięć \\
& & $\begin{array}{l}\text { A, że dziesięć } \\
\text { to max.,tak? } \\
\text { A dziewięć. }\end{array}$ & \\
translat & (Russ.) bis nein mit & $\begin{array}{l}\text { Acha, also zehn ist } \\
\text { null bis zehn (Poln.) } \\
\text { zehn stark (sic!) }\end{array}$ & $\begin{array}{l}\text { Maximum, ja? Also } \\
\text { neun. }\end{array}$ \\
& & & \\
\hline
\end{tabular}


[9]

\begin{tabular}{|llrl|} 
& 28 [01:46.1] & \multicolumn{1}{c|}{29 [01:48.1] } & 30 [01:53.3] \\
PN [v] & $\begin{array}{l}\text { Boli mocno, } \\
\text { Dziewięć. }\end{array}$ & bardzo. ((3s)) Lekarz! \\
NS3 [v] & & $\begin{array}{l}((\text { leises Lachen im Hintergr- } \\
\text { und) Hat er }\end{array}$ \\
translat & Es tut stark weh, sehr. Neun. Arzt! & \\
\hline
\end{tabular}

[10]

\begin{tabular}{|lllll|}
\hline KK [v] &.. & 31 [01:57.5] & 32 [02:04.3] 33 [02:04.9] \\
PN [v] & $\begin{array}{l}\text { ähm }((3 \mathrm{~s})) \mathrm{ma} \\
\text { Pan alergie? }\end{array}$ & Nie ma. \\
NS3 [v] & lek? & $\begin{array}{l}\text { Nie, nie mam. Nie wiem! Nie } \\
\text { wiem. Nie wiem. }\end{array}$ \\
translat & Medikament & $\begin{array}{l}\text { Haben Sie Al- } \\
\text { lergie? }\end{array}$ & $\begin{array}{l}\text { [KK: hat nicht] Nein, habe ich } \\
\text { nicht. Ich weiß nicht. }(2 x)\end{array}$ \\
\hline
\end{tabular}

[11]

\begin{tabular}{|lll|}
\hline KK [v] & Czy bierce leki? ((1s)) Pan lek? & $35[02: 13.4]$ \\
PN [v] & & $\begin{array}{l}\text { Biorę leki, biorę. Tak, biorę, biorę. Biorę. } \\
\text { Czasami, }\end{array}$ \\
translat & $\begin{array}{l}\text { Nehmen (sic!) Medikamente? Sie Medika- Ich nehme. Ja, ich nehme. Manchmal, } \\
\text { ment? }\end{array}$ \\
\hline
\end{tabular}

[12]

\begin{tabular}{|c|c|c|c|}
\hline & .. & 36 [02:22.2] & $37[02: 28.8]$ \\
\hline KK $[\mathbf{v}]$ & & $\begin{array}{l}((\text { leise })) \text { czasami. }((4 \mathrm{~s})) \\
\text { Jakie? }\end{array}$ & \\
\hline \multirow[t]{2}{*}{ PN [v] } & czasami. & & $\begin{array}{l}\text { Aa! Iboprofen, paraceta- } \\
\text { mol. Takie na ból. }\end{array}$ \\
\hline & manchmal & manchmal. Welche? & Solche gegen Schmerz. \\
\hline
\end{tabular}

[13]

\begin{tabular}{|c|c|c|}
\hline & 38 [02:36.5] & 39 [02:39.4] \\
\hline KK [v] & & $\begin{array}{l}\text { ok hmm }((4 s)) \text { habe ich schon gefragt. ja, ja. } \\
\text { ich will jetzt }\end{array}$ \\
\hline NS2 [v] & das heißt Schmerzmittel & \\
\hline
\end{tabular}


[14]

\begin{tabular}{|l|lll|}
\hline KK [v] & $\begin{array}{l}\text { fragen, wie oft er die } \\
\text { nimmt. }\end{array}$ & 403:00.1] & Y03:03.9] \\
PN [v] & $\begin{array}{l}\text { Boli mnie! Boli! ((Luft } \\
\text { schnappen)) }\end{array}$ & \\
translat & Tut mir weh. Tut weh! & (Eng.) Ja, ja. \\
\hline
\end{tabular}

[15]

\begin{tabular}{|c|c|c|c|c|c|c|}
\hline & . & 42 [03:06.8] & 43 [03:07.7] & 44 [03:08.6] & 45 [03:10.3] & 46 [03:12.7] \\
\hline KK [v] & $\begin{array}{l}\text { kommt. } \\
\text { ((lachen)) }\end{array}$ & & & & & $\begin{array}{l}\text { Kiedy byla } \\
\text { w }\end{array}$ \\
\hline NS2 [v] & & & Das stimmt. & & & \\
\hline NA [v] & & $\begin{array}{l}\text { aber nicht } \\
\text { jetzt }\end{array}$ & & & & \\
\hline NS3 [v] & & & & na ja & $\begin{array}{l}\text { ((unver- } \\
\text { ständlich)) }\end{array}$ & \\
\hline translat & & & & & & $\begin{array}{l}\text { Wann } \\
\text { waren Sie } \\
\text { (sic!) im }\end{array}$ \\
\hline
\end{tabular}

[16]

\begin{tabular}{|lllll|}
\hline KK [v] & szpitalu? & 47 [03:18.0] & $48[03: 23.4]$ & $49\left[03: 23.8^{*}\right]$ \\
PN [v] & & $\begin{array}{l}\text { Ma... } \\
\text { Byłem w szpitalu } \\
\text { ((Luftschnappen) })\end{array}$ & Ma Pan dokument? \\
translat & Kranknhaus? & $\begin{array}{l}\text { Ich war im Krank- } \\
\text { enhaus im letzten } \\
\text { Jahr. }\end{array}$ & Haben... & $\begin{array}{l}\text { Haben Sie Unter- } \\
\text { lagen? }\end{array}$ \\
\hline
\end{tabular}

[17]

\begin{tabular}{|lllll|}
\hline \multicolumn{1}{l|}{} & 50 [03:26.7] & 51 [03:32.3] & 52 [03:41.0] & 53 [03:41.6] \\
PN [v] & $\begin{array}{l}\text { Jaa. ((4s)) ähm } \\
\text { hmm Nie wiem. } \\
\text { Nie mam. Boli } \\
\text { mnie! Boli!! }\end{array}$ & & medi tlen \\
NA [v] & $\begin{array}{l}\text { Czy bierze pan } \\
\text { translat }\end{array}$ & $\begin{array}{l}\text { Ich weiß nicht. Ich } \\
\text { habe keine. Es tut } \\
\text { mir weh! Tut weh! }\end{array}$ & Nehmen Sie & leki? \\
\hline
\end{tabular}


Der Patient informiert darüber hinaus sachlich über die Beschwerden in der Zeile 3 mit der Äußerung „,Mam duszności“, welche zu den Assertiva zählt. Zu den Expressiva gehört seitens des Notfallsanitäters wiederum auch die in der Zeile 1 vorkommende Begrüßung „Dzień dobry!“. In Zeile 17 wird um Sauerstoff gebeten, somit gehört die Äußerung zu den Direktiva. Weitere Direktiva sind in den Fragen ${ }^{18}$ repräsentiert, beispielsweise nach dem Schmerz (Z. 3), den Medikamenten (Z. 12), einem Krankenhausbesuch (Z. 15-16) oder einer medizinischen Dokumentation (Z.16).

\subsection{Code-switching in der Notfallkommunikation}

Das Fallbeispiel zeigt interessante und unterrichtsrelevante Tendenzen, die insbesondere im Umgang des Notfallsanitäters mit den Gesprächsschritten des Patienten, der mit Nachdruck über seine Beschwerden berichtet, zum Vorschein kommen. Die Tendenzen sind dahingehend relevant, dass den Teilnehmenden anscheinend Antwortoptionen fehlen, um auf emotionale oder unverständliche Aussagen adäquat zu reagieren. Daher sollten mögliche Formulierungen zu einem weiteren Schwerpunkt des Unterrichts hinzugefügt werden. ${ }^{19}$

An dieser Stelle ist festzuhalten, dass bei notfallmedizinischen Sprachkursen in erster Linie Hören und Sprechen als Grundkompetenzen besonders unterstützt werden sollten. Hierzu könnte sowohl Einzel- als auch Gruppenarbeit helfen. Mögliche Übungen könnten schriftliche Zuordnungsaufgaben oder Hörverstehen mit vorgegebenen Antworten (Multiple-Choice) sein. Hier wäre auch Chunking (vgl. Roche 2013: 116) im Sinne von automatisierten Wiedergabe von nicht medizinbezogenen "Mehrworteinheiten, Formeln oder Redewendungen“ sinnvoll. Dies könnte durch fokussierte Sprechübungen geregelt werden, d.h. Erstellen von Dialogen nicht nur mit möglicher Auswahl an Formulierungen, aber mit fertigen Frage-Antwort-Sequenzen, die dann nicht als Option gesehen werden, sondern obligatorisch einzusetzen sind.

${ }^{18}$ Sprechakte sind ein Forschungsfeld, das im Rahmen des Artikels nicht ausschöpfend behandelt werden kann. Das gleiche betrifft die Klassifikationen der Sprechakte, die Fragehandlungen entweder den Direktiva oder den Erotetica zuordnen. Unter Erotetica sind nach Prokop (1995: 14) „[...] alle die Akte verstanden, mit denen sich der Sprecher an den Hörer wendet mit der Intention, eine Antwort zu bekommen. "Viele der Fragehandlungen könnten demzufolge tatsächlich als Erotetica bezeichnet werden. Aus forschungspraktischen Gründen werden diese in einer Kategorie als Direktiva zusammengefasst. An dieser Stelle soll mit Nachdruck auf die bereichernde Übersicht über die Sprechaktklassifikationen von Prokop (vgl. 1995: 47ff.) hingewiesen werden. Die Posener Linguistin hat sich um die Erforschung der Erotetica im Rahmen der deutsch-polnischen Gesprächsforschung verdient gemacht.

${ }^{19}$ Beispiele für die Formulierungen lauten: Jesteśmy przy panu / pani (Wir sind bei Ihnen), Zajmujemy się panem / panią, Lekarz już do pana / pani jedzie (Der Arzt ist schon unterwegs). 
Der Notfallsanitäter scheint sprachlich mit der Art und Weise, wie der Patient seine Beschwerden artikuliert, überfordert zu sein und wechselt vom Polnischen ins Englische und Deutsche. Vorher kam es bereits zum Sprachwechsel ins Russische. Folglich kommt es in dem Notfallgespräch zu Code-Switching ${ }^{20}$.

[12]

\begin{tabular}{|llll|} 
&.. & $36[02: 22.2]$ & $37[02: 28.8]$ \\
\hline KK $[\mathbf{v}]$ & & $(($ leise $))$ czasami. $((4 s))$ Jakie? & \\
PN $[\mathbf{v}]$ & czasami. & & Aau! Iboprofen, paracetamol. \\
& & & Takie na ból. \\
translat & manchmal & manchmal. Welche? & Solche gegen den Schmerz. \\
\hline
\end{tabular}

[13]

\begin{tabular}{|lll|} 
& 38 [02:36.5] & 39 [02:39.4] \\
KK [v] & & $\begin{array}{l}\text { ok hmm }((4))) \text { habe ich schon gefragt. ja, ja. } \\
\text { ich will jetzt }\end{array}$ \\
NS2 [v] & das heißt Schmerzmittel & \\
\hline
\end{tabular}

[14]

\begin{tabular}{|l|lll|}
\hline KK [v] & fragen, wie oft er die nimmt. & 40 [03:00.1] & 41 [03:03.9] \\
PN [v] & & $\begin{array}{l}\text { Boli mnie! Boli! ((Luft } \\
\text { schnappen)) }\end{array}$ & Yes, yes. Doktor \\
translat & & Tut mir weh! Tut weh! & (Eng.) Ja, ja. \\
\hline
\end{tabular}

[15]

\begin{tabular}{|c|c|c|c|c|c|c|}
\hline &.. & 42 [03:06.8] & 43 [03:07.7] & 44 [03:08.6] & 45 [03:10.3] & 46 [03:12.7] \\
\hline KK [v] & $\begin{array}{l}\text { kommt. } \\
\text { ((lachen)) }\end{array}$ & & & & & Kiedy byla w \\
\hline NS2 [v] & & & Das stimmt. & & & \\
\hline NA [v] & & $\begin{array}{l}\text { aber nicht } \\
\text { jetzt }\end{array}$ & & & & \\
\hline NS3 [v] & & & & na ja & $\begin{array}{l}\text { ((unver- } \\
\text { ständlich)) }\end{array}$ & $\begin{array}{l}\text { Wann waren } \\
\text { Sie im (sic!) }\end{array}$ \\
\hline
\end{tabular}

${ }^{20}$ Die Differenzierung in Code-Switching und Code-Mixing (vgl. Riehl 2014: 33) ist mir bekannt, jedoch eine vertiefte Auseinandersetzung ist mit diesen Begrifflichkeiten im Rahmen dieses Textes nicht möglich. Sowohl das intersententielle als auch das intrasententielle Sprachmischen wird im vorliegenden Artikel als Code-Switching bezeichnet. 
[16]

\begin{tabular}{|c|c|c|c|c|}
\hline &.. & 47 [03:18.0] & 48 [03:23.4] & 49 [03:23.8*] \\
\hline KK [v] & szpitalu? & & Мa... & Ma pan dokument? \\
\hline PN [v] & & $\begin{array}{l}\text { Byłem w szpitalu } \\
\text { w zeszłym roku. }\end{array}$ & $(($ Luftschnappen $))$ & \\
\hline translat & Krankenhaus? & $\begin{array}{l}\text { Ich war im Kranken- } \\
\text { haus im letzten Jahr. }\end{array}$ & Haben... & $\begin{array}{l}\text { Haben Sie die Unter- } \\
\text { lagen? }\end{array}$ \\
\hline
\end{tabular}

[17]

\begin{tabular}{|c|c|c|c|c|}
\hline & 50 [03:26.7] & 51 [03:32.3] & 52 [03:41.0] & 53 [03:41.6] \\
\hline KK [v] & & Jaa. ((4s)) ähm & & medi tlen \\
\hline PN [v] & $\begin{array}{l}\text { hmm Nie wiem. Nie } \\
\text { mam. Boli mnie! } \\
\text { Boli!!• }\end{array}$ & & & \\
\hline NA [v] & & & Czy bierze pan & leki? \\
\hline translat & $\begin{array}{l}\text { Ich weiß nicht. Ich } \\
\text { habe keine. Tut mir } \\
\text { weh! Tut weh!! }\end{array}$ & & Nehmen Sie & Medikamente? \\
\hline
\end{tabular}

In Zeile 13 wechselt der Notfallsanitäter ins Deutsche, da er den deutschsprachigen Kollegen erklärt, was er den Patienten genau gefragt hat (Zn. 13-14). An dieser Stelle ist die Sprachwahl in Abhängigkeit vom Gesprächspartner zu beobachten. In seinem darauffolgenden Turn beginnt der Notfallsanitäter auf Englisch (Z. 14), als der Patient über seine Schmerzen klagt, geht danach aber ins Deutsche über (Z. 15). In Zeile 17 scheint der Notfallsanitäter (Klaus Kleber) irritiert zu sein, was an der auf Deutsch realisierten und deutlich betonten Partikel ,ja" auszumachen ist.

Beim Code-Switching mit Kollegen trifft der Notfallsanitäter eine bewusste Wahl, da es ihm viel leichter fällt, auf Deutsch zu sprechen und dies natürlicher erscheint, da alle anwesenden Kollegen deutschsprachig sind. Darüber hinaus richtet sich die Information an andere Notfallsanitäter und nicht den Patienten, er ändert den Adressaten der Äußerung. Dies ist nach Blom und Gumperz (vgl. 1972: 424) sowie Gumperz (vgl. 1982: 92) als situatives Code-Switching zu bezeichnen. Die Anwendung des Polnischen wäre an dieser Stelle eher ungewöhnlich und nicht zielführend.

Nachdem der Notfallsanitäter den Kollegen das Gesagte verdeutlicht hat, fängt er an, Englisch zu sprechen: "Yes, yes“. Der Notfallsanitäter hat sich vorher mit seinen Kollegen auf Deutsch unterhalten, somit wäre eine Reaktion auf Deutsch denkbar. Andererseits wendet sich hier der Rettungssanitäter an den 
Patienten, der nur Polnisch versteht. Somit findet an dieser Stelle ein adressatenspezifischer Sprachwechsel statt. Auer (vgl. 1988: 196) spricht in einem solchen Fall von participant-related switching. Die Äußerung wird von den Kollegen aufgegriffen, die diese auf Deutsch ergänzen (Z. 15). Denkbar wäre auch, dass durch die Anwendung des Englischen die Rettungskraft eine neue Sprechphase markieren möchte oder unterstreicht, dass die Rettungskraft das Thema wechseln möchte (vgl. Auer 1988: 199) und somit ein discourse-related switching stattfindet.

Des Weiteren ist die Benutzung des Deutschen (Zn. 14-15 und 17) dahingehend für die Unterrichtseinheiten relevant, dass das Code-Switching hier eine referentielle Funktion (vgl. Appel \& Muysken 1987: 118ff.) übernimmt. Das lässt darauf schließen, dass die Kenntnisse im Polnisch nicht sehr ausgebaut sind oder dem Notfallsanitäter unter Stress leichter fällt, auf die im mentalen Lexikon gespeicherten Einheiten in der Muttersprache (hier: Deutsch) zurückzugreifen. Auch Auer (vgl. 1988: 196) sieht die Gründe für situatives Code-Switching in der Bevorzugung einer Sprache sowie fehlender Kompetenz in der anderen Sprache. Diese Perspektive lässt beide Interpretationen zu, die auch aus meiner Sicht zutreffen.

Das Code-Switching tritt ebenso beim Erfragen der Intensität der Schmerzen auf, obwohl der Rettungssanitäter hier ins Russische wechselt. Da der Lernende um die 40 Jahre alt ist und er als erste Fremdsprache Russisch deklarierte, ist dieser Sprachwechsel durch die typologische Nähe und einige Ähnlichkeiten zum Polnischen zu erklären (vgl. Rothman 2011: 112).

[3]

\begin{tabular}{|llll|}
\multicolumn{1}{l|}{} & 11 [00:20.3] & $12[00: 23.0]$ & $13[00: 28.2]$ \\
\hline KK [v] & y Co Pana boli? & & y Jak sylny to \\
PN [v] & & $\begin{array}{l}\text { ym uf Tu mnie boli. Tu tu } \\
\text { mnie boli. Mam duszności. }\end{array}$ & \\
translat & Was tut Ihnen weh? & $\begin{array}{l}\text { Hier tut mir weh. Hier } \\
\text { hier tut mir weh. Ich habe } \\
\text { Atemnot. }\end{array}$ & Wie stark (sic!) \\
& & & \\
\hline
\end{tabular}

[4]

\begin{tabular}{|lll|}
\hline KK [v] & zi zero do desjać ziro niet desjać sjlny & 14 [00:45.4] \\
PN [v] & & Może pan powtórzyć ((3.5s)) Może pan \\
translat & $\begin{array}{l}\text { (Russ.) von null bis zehn zehn nicht zehn } \\
\text { stark(sic!) }\end{array}$ & Können Sie wiederholen. Können Sie \\
\hline
\end{tabular}


[7]

\begin{tabular}{|c|c|c|c|c|c|c|}
\hline &.. & 20 [01:18.2] & 21 [01:24.0] & $22[01: 26.0]$ & 23 [01:26.2] & 24 [01:27.2] \\
\hline KK [v] & & e Jak sylny? & & Jak silny? ja! & & \\
\hline PN [v] & boli. & & Aa, jak silny? & & A, jak silny! & $\begin{array}{l}\text { A, jak boli tu } \\
\text { tak? Mocno. } \\
\text { Bardzo. }\end{array}$ \\
\hline translat & Tut weh. & $\begin{array}{l}\text { Wie stark } \\
\text { (sic!)? }\end{array}$ & & Wie stark? & $\begin{array}{l}\text { Acha, wie } \\
\text { stark! }\end{array}$ & $\begin{array}{l}\text { Acha, wie } \\
\text { stark tut } \\
\text { hier weh, ja? } \\
\text { Stark. Sehr. }\end{array}$ \\
\hline
\end{tabular}

[8]

\begin{tabular}{|c|c|c|c|}
\hline & 25 [01:31.9] & 26 [01:40.5] & 27 [01:45.6] \\
\hline KK [v] & $\begin{array}{l}\text { do ne s ziro do desjać. } \\
\text { desjąć sylny? }\end{array}$ & & djewięć \\
\hline PN [v] & & $\begin{array}{l}\text { A, że dziesięć to max.,tak? } \\
\text { A dziewięć. }\end{array}$ & \\
\hline translat & $\begin{array}{l}\text { (Russ.) bis null (sic!) } \\
\text { bis zehn (Poln.) zehn } \\
\text { stark?(sic!) }\end{array}$ & $\begin{array}{l}\text { Acha, zehn bedeutet Max- } \\
\text { imum, ja? Also neun. }\end{array}$ & neun (sic!) \\
\hline
\end{tabular}

Der Notfallsanitäter fragt nach den Schmerzen auf einer Skala von 0 bis 10 . Dabei verwendet er die Kardinalzahlen auf Russisch und auf Englisch. Er verwechselt ebenso die polnische Negationspartikel nie mit ihrem russischen Pendant net. Dem Notfallsanitäter fällt die Verwechslung der Sprachen anscheinend gar nicht auf, vielmehr scheint er mit der Bitte des Patienten zur Wiederholung überfordert zu sein. Der Patient reagiert höchstwahrscheinlich so, weil ein wichtiges Lexem in der Frage, nämlich das Signalwort ból (der Schmerz), fehlt. Es zeigt sich, wie stark zuerst gelernte Fremdsprachen und / oder typologisch der Zielsprache nahe stehende zuvor gelernte Fremdsprachen den Spracherwerb neuer Sprachen beeinflussen können. Dies ist für den Unterricht des Polnischen ebenso positiv zu werten, da Vergleiche z.B. in Bezug auf Zahlen getätigt werden können und die Zahlen sich dadurch leichter lernen lassen.

\section{ZUSAMMENFASSUNG UND AUSBLICK}

Das analysierte Fallbeispiel notfallmedizinischer Kommunikation zum Thema Atemnot lässt auf unterrichtsrelevante Tendenzen schließen.

Erfragen möglicher Allergien, Medikamenteneinnahme und Vorerkrankungen, Erfragen eines vergangenen Krankenhausbesuchs sind Sprechhandlungen, 
die anhand des Transkripts ausgemacht werden konnten. Themenbereiche werden für Anamnese zwar vorgegeben, aber die Art und Weise, auf welche die Abfrage stattfinden soll, ist nicht vordefiniert, erst recht nicht in der Fremdsprache, die im Rahmen eines Intensivsprachkurses erlernt wird. Somit lassen sich die in der Analyse erfassten Äußerungen in ihrer kontextgebundenen Ausprägung sinnvoll und für den Sprachunterricht produktiver betrachten.

Die Äußerungen lassen sich in folgende Sprechakte gruppieren: Assertiva, Direktiva und Expressiva. Die Expressiva werden in dem Gespräch vor allem vom Patienten produziert. Diese sind dahingehend unterrichtsrelevant, dass die Fremdsprachenlernenden die Antworten der Patienten auf Polnisch verstehen und auf sie adäquat reagieren sollten, auch wenn die Patienten ihren Aussagen eine emotionale Komponente hinzufügen. Aus der Untersuchung geht hervor, dass die Antworten des Patienten mehrheitlich verstanden werden, jedoch die Reaktion auf diese nicht immer adäquat ist.

Auf die Forschungsfrage zurückkommend - um die Situation kommunikativ erfolgreich zu meistern - müssen von den Fremdsprachenlernenden nicht medizinisch signifikante Chunks beherrscht werden. Diese festen Wendungen würden dann einerseits authentische Dynamik des Gesprächs gewährleisten, andererseits zur Beruhigung des Patienten sowie Stärkung der Selbstsicherheit des Sprechenden führen. Auch Anhand von Code-Switching-Sequenzen konnte die Notwendigkeit einer Modifikation und Erweiterung des Konzepts für den sprachpraktischen Unterricht ausgemacht werden. Es handelt sich um Reaktionen auf sprachliches Handeln des Patienten (vor allem Expressiva), die nicht direkt mit der Anamnese zu tun haben. Es geht größtenteils um das Beruhigen des Patienten, den Umgang mit Bitten um Wiederholung oder das Eingehen auf schmerzbedingte und schmerzbezogene Äußerungen. Die Wendungen können als Chunks bei Gruppenaufgaben geübt werden oder bei Einzelarbeit mit Hörverstehen gestärkt werden. ${ }^{21}$ Auch einfaches im Chor wiederholen kann beim automatisierten Einsetzen solcher Chunks helfen. Somit werden die Lernenden befähigt, ihre Antworten auf Polnisch und nicht auf Deutsch oder Englisch zu formulieren.

Wichtig für den Unterricht ist ebenso die sprachliche Realisierung der Fragen. $\mathrm{Zu}$ den am häufigsten produzierten Fragetypen gehören die Informationsfragen, die in der Kernphase des Notfallgesprächs angewandt werden. Sie sind von den Lernenden ausreichend verinnerlicht worden. Darüber hinaus wird die Relevanz der aus wenigen morphosyntaktischen Elementen bestehenden Fragen für die notfallmedizinische Kommunikation und somit für den Unterricht noch deut-

\footnotetext{
${ }^{21}$ Beispiele für die hier thematisierten Chunks lauten: Jak silny to ból? (Wie stark sind die Schmerzen?), Podaliśmy już leki! (Wir haben schon Medikamende verabreicht!), Proszę powtórzyć! (Bitte wiederholen!), Proszę wolniej! (Bitte langsamer!).
} 
licher. Es ist dahingehend ein Erkenntnisgewinn, dass im Sprachkurs mehrere Varianten von Fragenformulierungen zum gleichen Sachverhalt vorgestellt und geübt wurden. Während des Rollenspiels wurden elliptische Formulierungen benutzt. Die Lernenden haben wahrscheinlich die Formulierungen mit der für sie am einfachsten erscheinenden Aussprache gewählt. Bei der Weiterentwicklung des Fachsprachenkurses können die Folgegruppen noch zielgerichteter auf ihre fremdsprachigen Einsätze vorbereitet werden. Darüber hinaus wäre es sinnvoll weiterhin die Rollenspiele (Kurzsimulationen) im Rahmen des Unterrichts fortzuführen, da die Sprachproduktion eine ertragreichere als bei anderen Übungstypen ist. Die Kurzsimulationen sind förderlich, obwohl die Sprachprodukte einen elliptischen Charakter haben oder von Code Switching geprägt sind.

Zwar gewähren die Ergebnisse interessante Einblicke und weisen auf nötige Modifikationen in der Gestaltung des Sprachunterrichts hin, aber es bleibt einschränkend zu bemerken, dass ein Fallbeispiel nur bedingt repräsentativ ist.

\section{LITERATURVERZEICHNIS}

Albert, R. / Koster, C.J. (2002). Empirie in Linguistik und Sprachlehrforschung: ein methodologisches Arbeitsbuch. Tübingen: Narr.

Appel, R. / Muysken, P. (1987). Language contact and bilingualism. London: Arnold.

Auer, P. (1988). A conversation analytic approach to code-switching and transfer. In: M. Heller (Hrsg.), Codeswitching: Anthropological and sociological perspectives (S. 187-213). Berlin: Mouton de Gruyter.

Bechmann, S. (2014). Sprache und Medizin: interdisziplinäre Beiträge zur medizinischen Sprache und Kommunikation. Berlin: Frank \& Timme Verlag für wissenschaftliche Literatur.

Blom, J.-P. / Gumperz, J.J. (1972). Social meaning in linguistic structure: Code-switching in Norway. In: J.J. Gumperz / D. Hymes (Hrsg.), Directions in sociolinguistics (S. 407-434). New York: Holt, Rinehart \& Winston.

Brehmer, B. (2018). Polnisch als Nachbarsprache in Deutschland. Polski w Niemczech - Pismo Federalnego Zwiazku Nauczycieli Języka Polskiego / Polnisch in Deutschland - Zeitschrift der Bundesvereinigung der Polnischkräfte. Numer specjalny / Sondernummer, 21-36.

Gębal, P.E. (2016). Od dydaktyki tekstów specjalistycznych do dydaktyzacji potrzeb i działań komunikacyjnych. Kierunki i koncepcje glottodydaktyki specjalistycznej. Acta Universitatis Lodziensis. Ksztatcenie Polonistyczne Cudzoziemców, 23, 19-33.

Gumperz, J.J. (1982). Discourse strategies. Cambridge: Cambridge University Press.

Hryniewicz, J. / Lisek, G. (2019). Nauka języka sąsiada: od kształcenia przedszkolnego do zawodowego. Rozwiązania dydaktyczne i organizacyjne na Pomorzu Przednim. In: K. Zioło-Pużuk (Hrsg.), Panorama glottodydaktyki polonistycznej. Wyzwania, pytania, kierunki (S. 149-162). Warszawa: Wydawnictwo UKSW.

Illés, B. / Kegyes, I. (2019). Die Rolle der Simulation im Fachsprachenunterricht. In: E. Kegyes / R. Kriston / M. Schönenberger (Hrsg.), Sprachen, Literaturen und Kulturen im Kontakt. Beiträge der 25. Linguistik- und Literaturtage, Miskolc/Ungarn, 2017 (S. 117-126). Hamburg: Verlag Dr. Kovač. 
Kiesendahl, J. (2011). Status und Kommunikation: ein Vergleich von Sprechhandlungen in universitären E-Mails und Sprechstundengesprächen. Berlin: Schmidt.

Klčova, R. (2019). Die simulierte Hauptverhandlung im Spannungsfeld von linguistischer und juristischer Dimension. In: E. Kegyes / R. Kriston / M. Schönenberger (Hrsg.), Sprachen, Literaturen und Kulturen im Kontakt. Beiträge der 25. Linguistik- und Literaturtage, Miskolc / Ungarn, 2017 (S. 127-132). Hamburg: Verlag Dr. Kovač.

Mroczynski, R. (2014). Gesprächslinguistik: eine Einführung. Tübingen: Narr.

Prokop, I. (1995). Erotetische Sprechakte im Deutschen und im Polnischen anhand natürlicher Gespräche. Poznań: Wydawnictwo Naukowe UAM.

Riehl, C.M. (2014). Mehrsprachigkeit: eine Einführung. Darmstadt: WBG.

Roche, J. (2013). Fremdsprachenerwerb - Fremdsprachendidaktik. Tübingen, Basel: Francke.

Rothmann, J. (2011). L3 syntactic transfer selectivity and typological determinacy: The typological primacy model. Second Language Research, 27, 107-127.

Searle, J.R. (1997). Sprechakte: ein sprachphilosophischer Essay. Frankfurt a. M.: Suhrkamp.

Spranz-Fogasy, T. / Becker, M. (2015). Beschwerdenexploration und Diagnosemitteilung im ärztlichen Erstgespräch. In: A. Busch / T. Spranz-Fogasy (Hrsg.), Handbuch Sprache in der Medizin (S. 93-115). Berlin, Boston: De Gruyter.

Stemmler, J. / Hecker, U. (2017). Notfallkommando Kommunikation in Notfallsituationen für Gesundheitsberufe. Berlin, Heidelberg: Springer.

Szerszeń, P. (2014). Platformy (glotto)dydaktyczne. Ich implementacja w uczeniu specjalistycznych języków obcych. Warszawa: Wydawnictwo Naukowe Instytutu Kulturologii i Lingwistyki Antropocentrycznej.

Thun von, Sch. (1998). Miteinander reden 1. Reinbek bei Hamburg: Rowohlt-Taschenbuch-Verl.

Zschäbitz, E. (2013). Web 2.0 to nic strasznego. Rozwijanie kompetencji językowych za pomocą internetowych narzędzi edukacyjnych. Polski w Niemczech - Pismo Federalnego Zwiazku Nauczycieli Jezzyka Polskiego / Polnisch in Deutschland - Zeitschrift der Bundesvereinigung der Polnischkräfte, $1,44-52$.

Received: 21.04.2020; revised: 08.07.2020

GRZEGORZ LISEK

Universität Greifswald

grzegorz.lisek@uni-greifswald.de

ORCID: 0000-0003-1613-3855 
